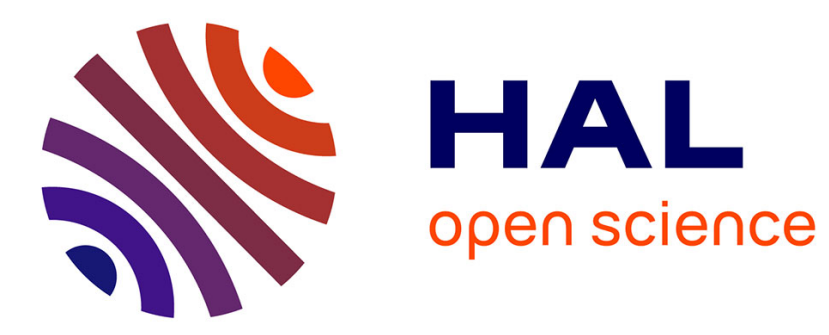

\title{
Two-temperature homogenized eigenfunctions of conduction through domains with jump interfaces
}

Isabelle Gruais, Dan Poliševski, Alina Stefan

\section{To cite this version:}

Isabelle Gruais, Dan Poliševski, Alina Stefan. Two-temperature homogenized eigenfunctions of conduction through domains with jump interfaces. Applicable Analysis, 2020, 99 (13), pp.2361-2370. 10.1080/00036811.2018.1563292 . hal-01874134

\section{HAL Id: hal-01874134 \\ https://hal.science/hal-01874134}

Submitted on 14 Sep 2018

HAL is a multi-disciplinary open access archive for the deposit and dissemination of scientific research documents, whether they are published or not. The documents may come from teaching and research institutions in France or abroad, or from public or private research centers.
L'archive ouverte pluridisciplinaire HAL, est destinée au dépôt et à la diffusion de documents scientifiques de niveau recherche, publiés ou non, émanant des établissements d'enseignement et de recherche français ou étrangers, des laboratoires publics ou privés. 


\title{
Two-temperature homogenized eigenfunctions of conduction through domains with jump interfaces
}

\author{
Isabelle Gruais, Dan Poliševski and Alina Ştefan
}

\begin{abstract}
In this paper we study the asymptotic behavior of the eigenvalue problem solutions of the conduction process in an $\varepsilon$-periodic domain formed by two components separated by a first-order jump interface. We prove that when $\varepsilon \rightarrow 0$ the limits of the eigenvalues and eigenfunctions of this problem verify a certain (effective) two-temperature eigenvalue problem. Moreover, we show that the effective eigenvalue problem has only eigenvalues which come from the homogenization process.
\end{abstract}

Keywords: interfacial jump, conduction, eigenvalue, two-scale convergence, homogenization

MSC 2010: 35B27, 35P15, 49R05, 74A50, 80M40

\section{Introduction}

During the last decades there was a steady interest for the homogenization of problems with interfacial thermal barriers (see [2], [14], [7], [5]) or equivalent (see [13], [11], [8], [15]). Meanwhile, it was also studied the asymptotic behavior of the eigenvalue problems in $\varepsilon$-periodic domains (see $[12]$ ), even for $\varepsilon$-periodically perforated domains (see [17]), where the key ingredient was the prolongation operator which was introduced by [6].

Here we continue our works [9], [10] and [16] by studying the asymptotic behavior of the eigenvalue problem solutions of the conduction process in an $\varepsilon$-periodic domain formed by two components separated by a first-order jump interface. We prove that when $\varepsilon \rightarrow 0$ the limits of the eigenvalues and eigenfunctions of this problem verify a certain (effective) two-temperature eigenvalue problem. Moreover, we show that the effective eigenvalue problem has only eigenvalues which come from the homogenization process. Our key ingredient is a pair of prolongation operators, corresponding to each component of the domain. Otherwise, we mainly follow the methods of the two-scale theory (see [1]). We have to remark that the procedure presented here can straightforwardly be generalized to any $\mathrm{n}$-component $\varepsilon$-periodic domain with interfacial jumps of the first order. 
The paper is organized as follows: in Section 2 we study the eigenvalue problem in the $\varepsilon$-periodic domain; in Section 3 we present the key prolongation operators, the a priori estimates and the specific compactness results; Section 4 is devoted to the derivation of the effective eigenvalue problem and its connection with the homogenization process.

\section{The eigenvalue problem}

Let $\Omega$ be an open connected bounded set in $\mathbb{R}^{N}(N \geq 3)$, locally located on one side of the boundary $\partial \Omega$, a Lipschitz manifold composed of a finite number of connected components. For any $\varepsilon \in(0,1), \Omega$ has two $\varepsilon$-periodically ditributed components. For convenience, the periodicity is described by using the cube $Y=(0,1)^{N}$, as follows:

Let $Y_{a} \subset \subset Y$ be a Lipschitz open set such that $Y_{b}=Y \backslash \bar{Y}_{a}$ is connected. For any $\varepsilon \in(0,1)$ we denote

$$
\mathbb{Z}_{\varepsilon}=\left\{k \in \mathbb{Z}^{N}: \varepsilon k+\varepsilon Y \subseteq \Omega\right\} .
$$

The two $\varepsilon$-periodic components of $\Omega$ are defined by:

$$
\begin{gathered}
\Omega_{\varepsilon a}=\operatorname{int}\left(\bigcup_{k \in \mathbb{Z}_{\varepsilon}}\left(\varepsilon k+\varepsilon \bar{Y}_{a}\right)\right) \\
\Omega_{\varepsilon b}=\Omega \backslash \bar{\Omega}_{\varepsilon a} .
\end{gathered}
$$

Denoting $\Gamma:=\partial Y_{a}=\partial Y_{a} \cap \partial Y_{b}$, the interface between $\Omega_{\varepsilon a}$ and $\Omega_{\varepsilon b}$ have the property:

$$
\Gamma_{\varepsilon}:=\bigcup_{k \in \mathbb{Z}_{\varepsilon}}(\varepsilon k+\varepsilon \Gamma)=\partial \Omega_{\varepsilon a}=\partial \Omega_{\varepsilon a} \cap \partial \Omega_{\varepsilon b} .
$$

Let us remark that $\Omega_{\varepsilon b}$ is connected and all the boundaries are at least locally Lipschitz. Also, the inward normal on $\partial Y_{a}$, denoted by $\nu$, has the property

$$
\nu^{\varepsilon}(x)=\nu\left(\left\{\varepsilon^{-1} x\right\}\right), \quad \forall x \in \Gamma_{\varepsilon},
$$

where $\left\{\varepsilon^{-1} x\right\}$ is formed by the fractional parts of the components of $\varepsilon^{-1} x$.

We have to introduce the Hilbert space

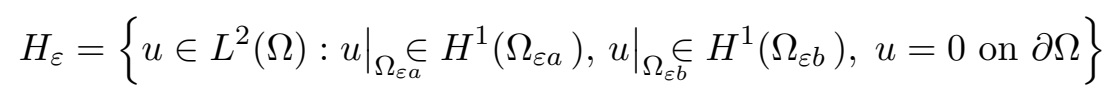

endowed with the scalar product

$$
(u, v)_{H_{\varepsilon}}=\int_{\Omega_{\varepsilon a}} \nabla u \nabla v+\int_{\Omega_{\varepsilon b}} \nabla u \nabla v+\varepsilon \int_{\Gamma_{\varepsilon}}[u][v],
$$

where $[u]=\gamma_{\varepsilon a} u-\gamma_{\varepsilon b} u$ and $\gamma_{\varepsilon a} u, \gamma_{\varepsilon b} u$ are the traces of $u$ on $\Gamma_{\varepsilon}$ defined in $H^{1}\left(\Omega_{\varepsilon a}\right)$ and $H^{1}\left(\Omega_{\varepsilon b}\right)$, respectively.

Our domain has the following well-known property [8]: 
Lemma 2.1. For any $v \in H_{\varepsilon}$ there exists $C>0$, independent of $\varepsilon$, such that

$$
\begin{gathered}
|v|_{L^{2}\left(\Omega_{\varepsilon b}\right)} \leq C|\nabla v|_{L^{2}\left(\Omega_{\varepsilon b}\right)}, \\
\varepsilon^{1 / 2}\left|\gamma_{\varepsilon \alpha} v\right|_{L^{2}\left(\Gamma_{\varepsilon}\right)} \leq C\left(|v|_{L^{2}\left(\Omega_{\varepsilon_{\alpha}}\right)}+\varepsilon|\nabla v|_{L^{2}\left(\Omega_{\varepsilon_{\alpha}}\right)}\right), \alpha \in\{a, b\}, \\
|v|_{L^{2}\left(\Omega_{\varepsilon_{a}}\right)} \leq C\left(\varepsilon^{1 / 2}\left|\gamma_{\varepsilon a} v\right|_{L^{2}\left(\Gamma_{\varepsilon}\right)}+\varepsilon|\nabla v|_{L^{2}\left(\Omega_{\varepsilon_{a}}\right)}\right) .
\end{gathered}
$$

Remark 2.1. Taking in account the $L^{2}$-norm of the jump on $\Gamma_{\varepsilon}$ the results of the previous Lemma have important consequences:

$$
\begin{gathered}
\varepsilon^{1 / 2}|[v]|_{L^{2}\left(\Gamma_{\varepsilon}\right)} \leq C\left(|v|_{L^{2}(\Omega)}+\varepsilon|\nabla v|_{L^{2}\left(\Omega_{\varepsilon a}\right)}+\varepsilon|\nabla v|_{L^{2}\left(\Omega_{\varepsilon b}\right)}\right), \\
|v|_{L^{2}\left(\Omega_{\varepsilon a}\right)} \leq C|v|_{H_{\varepsilon}}, \forall v \in H_{\varepsilon} .
\end{gathered}
$$

Next, we introduce the data of our problem: the transmission factor $h^{\varepsilon}(x)=h(x / \varepsilon)$ and the symmetric conductivities $a_{i j}^{\varepsilon}(x)=a_{i j}(x / \varepsilon)$ and $b_{i j}^{\varepsilon}(x)=b_{i j}(x / \varepsilon)$, where $h, a_{i j}$ and $b_{i j}$ belong to $L_{p e r}^{\infty}(Y)$ and have the property that there exists $\delta>0$ such that

$$
\begin{gathered}
h \geq \delta \text {, a.e. on } Y, \\
a_{i j} \xi_{j} \xi_{i} \geq \delta \xi_{i} \xi_{i} \text { and } b_{i j} \xi_{j} \xi_{i} \geq \delta \xi_{i} \xi_{i}, \quad \forall \xi \in \mathbb{R}^{N}, \text { a.e. on } Y \text {. }
\end{gathered}
$$

We consider the following eigenvalue problem:

Find $\lambda^{\varepsilon} \in \mathbb{R}^{*}$ such that $\exists u^{\varepsilon} \in H_{\varepsilon} \backslash\{0\}$ verifying the equations

$$
\begin{aligned}
& -\operatorname{div}\left(a^{\varepsilon} \nabla u^{\varepsilon}\right)=\lambda^{\varepsilon} u^{\varepsilon}, \quad \text { in } \Omega_{\varepsilon a}, \\
& -\operatorname{div}\left(b^{\varepsilon} \nabla u^{\varepsilon}\right)=\lambda^{\varepsilon} u^{\varepsilon}, \quad \text { in } \Omega_{\varepsilon b},
\end{aligned}
$$

and the transmission conditions

$$
a_{i j}^{\varepsilon} \frac{\partial u^{\varepsilon}}{\partial x_{j}} \nu_{i}^{\varepsilon}=b_{i j}^{\varepsilon} \frac{\partial u^{\varepsilon}}{\partial x_{j}} \nu_{i}^{\varepsilon}=\varepsilon h^{\varepsilon}\left(\gamma_{\varepsilon a} u^{\varepsilon}-\gamma_{\varepsilon b} u^{\varepsilon}\right) \text { on } \Gamma_{\varepsilon} .
$$

The variational formulation of the problem (15)-(17) is the following:

Find $\lambda^{\varepsilon} \in \mathbb{R}^{*}$ such that $\exists u^{\varepsilon} \in H_{\varepsilon} \backslash\{0\}$ verifying

$$
\begin{gathered}
G_{\varepsilon}\left(u^{\varepsilon}, v\right):=\int_{\Omega_{\varepsilon a}} a_{i j}^{\varepsilon} \frac{\partial u^{\varepsilon}}{\partial x_{j}} \frac{\partial v}{\partial x_{i}}+\int_{\Omega_{\varepsilon b}} b_{i j}^{\varepsilon} \frac{\partial u^{\varepsilon}}{\partial x_{j}} \frac{\partial v}{\partial x_{i}}+\varepsilon \int_{\Gamma_{\varepsilon}} h^{\varepsilon}\left[u^{\varepsilon}\right][v]=\lambda^{\varepsilon}\left(u^{\varepsilon}, v\right), \\
\forall v \in H_{\varepsilon}
\end{gathered}
$$

where $(\cdot, \cdot)$ denotes the inner product in $L^{2}(\Omega)$.

Using the procedure of [3], we introduce the operator $T^{\varepsilon} \in \mathcal{L}\left(L^{2}(\Omega), H_{\varepsilon}\right)$ by denotig with $T^{\varepsilon}(u)$, for $u \in L^{2}(\Omega)$, the unique solution of the problem

$$
\int_{\Omega_{\varepsilon a}} a_{i j}^{\varepsilon} \frac{\partial T^{\varepsilon}(u)}{\partial x_{j}} \frac{\partial v}{\partial x_{i}}+\int_{\Omega_{\varepsilon b}} b_{i j}^{\varepsilon} \frac{\partial T^{\varepsilon}(u)}{\partial x_{j}} \frac{\partial v}{\partial x_{i}}+\varepsilon \int_{\Gamma_{\varepsilon}} h^{\varepsilon}\left[T^{\varepsilon}(u)\right][v]=\int_{\Omega} u v, \forall v \in H_{\varepsilon} .
$$


Defining $\tilde{T}^{\varepsilon}: L^{2}(\Omega) \rightarrow L^{2}(\Omega)$ by $\tilde{T}^{\varepsilon}=J^{\varepsilon} \circ T^{\varepsilon}$, where $J^{\varepsilon}$ is the inclusion of $H_{\varepsilon}$ into $L^{2}(\Omega)$, we see that the eigenvalue problem (18) is equivalent to the eigenvalue problem

$$
\tilde{T}^{\varepsilon} u^{\varepsilon}=\mu^{\varepsilon} u^{\varepsilon}, \text { via } \mu^{\varepsilon}=\frac{1}{\lambda^{\varepsilon}} .
$$

Lemma 2.2. The inclusion $J^{\varepsilon}: H_{\varepsilon} \rightarrow L^{2}(\Omega)$ is a compact operator.

Proof. As for any $v \in H_{\varepsilon}$ we have

$$
\left|J^{\varepsilon} v\right|_{L^{\varepsilon}(\Omega)}=|v|_{\Omega_{\varepsilon a}}+|v|_{\Omega_{\varepsilon b}} \leq C|v|_{H_{\varepsilon}},
$$

is sufficient to prove that the bounded sequences from $H_{\varepsilon}$ contain a convergent subsequence in $L^{2}(\Omega)$.

Let $\left\{v_{n}\right\}_{n}$ a bounded sequence in $H_{\varepsilon}$. We note $v_{n}^{a}=\left.v_{n}\right|_{\Omega_{\varepsilon a}}$ and $v_{n}^{b}=$ $\left.v_{n}\right|_{\Omega_{\varepsilon b}}$. Since $\left\{v_{n}^{a}\right\}_{n}$ is a bounded sequence in $H^{1}\left(\Omega_{\varepsilon a}\right)$, from the Rellich's theorem there exist $v^{a} \in H^{1}\left(\Omega_{\varepsilon a}\right)$ and a subsequence, still denoted by $\{n\}$, such that

$$
v_{n}^{a} \rightarrow v^{a} \text { strongly in } L^{2}\left(\Omega_{\varepsilon a}\right) .
$$

Further, $\left\{v_{n}^{b}\right\}_{n}$ being bounded in $H^{1}\left(\Omega_{\varepsilon b}\right)$, again the Rellich's theorem implies the existence of some $v^{b} \in H^{1}\left(\Omega_{\varepsilon b}\right)$ such that on a sub-subsequence it holds

$$
v_{n}^{b} \rightarrow v^{b} \text { strongly in } L^{2}\left(\Omega_{\varepsilon b}\right) .
$$

It follows that

$$
v_{0}=\left\{\begin{array}{ll}
v^{a} & \text { in } \Omega_{\varepsilon a}, \\
v^{b} & \text { in } \Omega_{\varepsilon b}
\end{array} \Rightarrow v_{0} \in H_{\varepsilon} \subset L^{2}(\Omega) .\right.
$$

The proof is completed as

$$
\left|v_{n}-v_{0}\right|_{L^{2}(\Omega)}^{2}=\left|v_{n}^{a}-v^{a}\right|_{L^{2}\left(\Omega_{\varepsilon a}\right)}^{2}+\left|v_{n}^{b}-v^{b}\right|_{L^{2}\left(\Omega_{\varepsilon b}\right)}^{2} \rightarrow 0 .
$$

We see now that $\tilde{T}^{\varepsilon}$ is a self-adjoint, compact operator in $L^{2}(\Omega)$ and recalling for instance [4] it follows that there exist $\left\{\lambda_{k}^{\varepsilon}\right\}_{k}$, eigenvalues of the problem (18), with the property

$$
0<\lambda_{1}^{\varepsilon} \leq \lambda_{2}^{\varepsilon} \leq \ldots \rightarrow \infty
$$

and $\left\{u_{k}^{\varepsilon}\right\}_{k}$, the corresponding eigenfunctions, which are complete and orthonormal in $L^{2}(\Omega)$.

In the following sections we shall study the behaviour of $\left(\lambda_{k}^{\varepsilon}, u_{k}^{\varepsilon}\right)$ when $\varepsilon \rightarrow 0$. 


\section{A priori estimates}

We begin this section by proving the boundedness of $\left\{\lambda_{k}^{\varepsilon}\right\}_{\varepsilon}$, the eigenvalues of (18). For every $k \in \mathbb{N}$ let us denote

$$
H_{\varepsilon, k}=\left\{S \text { subspace of } H_{\varepsilon}, \operatorname{dim} S=k\right\} .
$$

Applying the Minimum-maximum principle (see [3]), $\lambda_{k}^{\varepsilon}$ can be estimated via the Rayleigh quotient:

$$
\begin{gathered}
\lambda_{k}^{\varepsilon}=\min _{S \in H_{\varepsilon, k}} \max _{v \in S} \frac{G_{\varepsilon}(v, v)}{|v|_{L^{2}(\Omega)}^{2}} \leq \\
\leq C \min _{S \in H_{\varepsilon, k}} \max _{v \in S} \frac{|\nabla v|_{L^{2}\left(\Omega_{\varepsilon a}\right)}^{2}+|\nabla v|_{L^{2}\left(\Omega_{\varepsilon b}\right)}^{2}+\varepsilon|[v]|_{L^{2}\left(\Gamma_{\varepsilon}\right)}^{2} .}{|v|_{L^{2}(\Omega)}^{2}} .
\end{gathered}
$$

In order to further estimate $\lambda_{k}^{\varepsilon}$ with respect to $\varepsilon$ we introduce two prolongation operators.

First, for any $v \in H^{1}\left(Y_{a}\right)$ let $w \in H^{1}\left(Y_{b}\right)$ be the only solution of the Dirichlet problem:

$$
\begin{gathered}
-\nabla w=0 \text { in } Y_{b}, \\
w=0 \text { on } \partial Y, \quad w=v \text { on } \Gamma .
\end{gathered}
$$

We have to introduce here $P_{a}(v) \in H^{1}(Y)$ by

$$
P_{a}(v)= \begin{cases}v & \text { in } Y_{a} \\ w & \text { in } Y_{b}\end{cases}
$$

It has the property

$$
\left|P_{a}(v)\right|_{H^{1}(Y)} \leq C|v|_{H^{1}\left(Y_{a}\right)} .
$$

Denoting $u_{k}^{\varepsilon}(y):=u(\varepsilon k+\varepsilon y)$, for any $k \in \mathbb{Z}_{\varepsilon}, y \in Y_{a}$ and $u \in H^{1}\left(\Omega_{\varepsilon a}\right)$, we define our first prolongation operator, $P_{a}^{\varepsilon}: H^{1}\left(\Omega_{\varepsilon a}\right) \rightarrow H_{0}^{1}(\Omega)$, by

$$
P_{a}^{\varepsilon}(u)(x)= \begin{cases}u(x) & \text { for } x \in \Omega_{\varepsilon a} \\ P_{a}\left(u_{k}^{\varepsilon}\right)\left(\left\{\frac{x}{\varepsilon}\right\}\right) & \text { for } x \in\left(\varepsilon k+\varepsilon Y_{b}\right), k \in \mathbb{Z}_{\varepsilon} \\ 0 & \text { for } x \in \Omega \backslash \bigcup_{k \in \mathbb{Z}_{\varepsilon}}(\varepsilon k+\varepsilon \bar{Y})\end{cases}
$$

Rescaling (22) we easily obtain

$$
\left|P_{a}^{\varepsilon}(u)\right|_{H^{1}(\Omega)} \leq C\left(|u|_{L^{2}\left(\Omega_{\varepsilon a}\right)}+\varepsilon|\nabla u|_{L^{2}\left(\Omega_{\varepsilon a}\right)}\right), \quad \forall v \in H^{1}\left(\Omega_{\varepsilon a}\right) .
$$

Second, for any $v \in H^{1}\left(Y_{b}\right)$ let $w \in H^{1}\left(Y_{b}\right)$ be the only solution of the Dirichlet problem:

$$
-\nabla w=0 \text { in } Y_{a},
$$




$$
w=v \text { on } \Gamma \text {. }
$$

For this component we introduce $P_{b}(v) \in H^{1}(Y)$ by

$$
P_{b}(v)= \begin{cases}w & \text { in } Y_{a} \\ v & \text { in } Y_{b}\end{cases}
$$

which has a property similar to (22):

$$
\left|P_{b}(v)\right|_{H^{1}(Y)} \leq C|v|_{H^{1}\left(Y_{b}\right)} .
$$

Denoting $u_{k}^{\varepsilon}(y):=u(\varepsilon k+\varepsilon y)$, for any $k \in \mathbb{Z}_{\varepsilon}, y \in Y_{b}, u \in H^{1}\left(\Omega_{\varepsilon b}\right)$, $u=0$ on $\partial \Omega$, we define our second prolongation operator, $P_{b}^{\varepsilon}:\{u \in$ $H^{1}\left(\Omega_{\varepsilon b}\right), \mathrm{u}=0$ on $\left.\partial \Omega\right\} \rightarrow H_{0}^{1}(\Omega)$, by

$$
P_{b}^{\varepsilon}(u)(x)= \begin{cases}u(x) & \text { for } x \in \Omega_{\varepsilon b} \\ P_{b}\left(u_{k}^{\varepsilon}\right)\left(\left\{\frac{x}{\varepsilon}\right\}\right) & \text { for } x \in\left(\varepsilon k+\varepsilon Y_{a}\right), k \in \mathbb{Z}_{\varepsilon} .\end{cases}
$$

Rescaling (26) we get

$$
\begin{gathered}
\left|P_{b}^{\varepsilon}(u)\right|_{H^{1}(\Omega)} \leq C\left(|u|_{L^{2}\left(\Omega_{\varepsilon b}\right)}+\varepsilon|\nabla u|_{L^{2}\left(\Omega_{\varepsilon b}\right)}\right), \\
\forall u \in H^{1}\left(\Omega_{\varepsilon b}\right), \quad \mathrm{u}=0 \text { on } \partial \Omega .
\end{gathered}
$$

We can now estimate the terms of (19).

The first term gives:

$$
\frac{|\nabla v|_{L^{2}\left(\Omega_{\varepsilon a}\right)}^{2}}{|v|_{L^{2}(\Omega)}^{2}} \leq \frac{\left|\nabla P_{a}^{\varepsilon}(v)\right|_{L^{2}\left(\Omega_{\varepsilon a}\right)}^{2}}{\left|P_{a}^{\varepsilon}(v)\right|_{L^{2}(\Omega)}^{2}} \cdot \frac{\left|P_{a}^{\varepsilon}(v)\right|_{L^{2}(\Omega)}^{2}}{|v|_{L^{2}(\Omega)}^{2}}<C \frac{\left|P_{a}^{\varepsilon}(v)\right|_{L^{2}(\Omega)}^{2}}{|v|_{L^{2}(\Omega)}^{2}}
$$

the constant being the first eigenvalue of the problem (20)-(21). Using (23) we obtain

$$
\frac{|\nabla v|_{L^{2}\left(\Omega_{\varepsilon a}\right)}^{2}}{|v|_{L^{2}(\Omega)}^{2}}<C_{1}+\varepsilon^{2} C_{2} \frac{\int_{\Omega_{\varepsilon a}} a^{\varepsilon} \nabla v \nabla v}{|v|_{L^{2}(\Omega)}^{2}} .
$$

The second term gives:

$$
\frac{|\nabla v|_{L^{2}\left(\Omega_{\varepsilon b}\right)}^{2}}{|v|_{L^{2}(\Omega)}^{2}} \leq \frac{\left|\nabla P_{b}^{\varepsilon}(v)\right|_{L^{2}\left(\Omega_{\varepsilon b}\right)}^{2}}{\left|P_{b}^{\varepsilon}(v)\right|_{L^{2}(\Omega)}^{2}} \cdot \frac{\left|P_{b}^{\varepsilon}(v)\right|_{L^{2}(\Omega)}^{2}}{|v|_{L^{2}(\Omega)}^{2}}<C \frac{\left|P_{b}^{\varepsilon}(v)\right|_{L^{2}(\Omega)}^{2}}{|v|_{L^{2}(\Omega)}^{2}}
$$

the constant being the first eigenvalue of the problem (24)-(25). Using (27) we obtain

$$
\frac{|\nabla v|_{L^{2}\left(\Omega_{\varepsilon b}\right)}^{2}}{|v|_{L^{2}(\Omega)}^{2}}<C_{1}+\varepsilon^{2} C_{2} \frac{\int_{\Omega_{\varepsilon b}} b^{\varepsilon} \nabla v \nabla v}{|v|_{L^{2}(\Omega)}^{2}}
$$


The third term can be estimated from (11), that is

$$
\frac{|[v]|_{L^{2}\left(\Gamma_{\varepsilon}\right)}^{2}}{|v|_{L^{2}(\Omega)}^{2}}<C_{1}+\varepsilon^{2} C_{2} \frac{\int_{\Omega_{\varepsilon a}} a^{\varepsilon} \nabla v \nabla v}{|v|_{L^{2}(\Omega)}^{2}}+\varepsilon^{2} C_{2} \frac{\int_{\Omega_{\varepsilon b}} b^{\varepsilon} \nabla v \nabla v}{|v|_{L^{2}(\Omega)}^{2}} .
$$

Adding the estimates (28)-(30) we get

$$
\lambda_{k}^{\varepsilon} \leq C_{1}+\varepsilon^{2} C_{2} \lambda_{k}^{\varepsilon}
$$

which obviously implies that $\lambda_{k}^{\varepsilon}$ is bounded for sufficiently small $\varepsilon$.

Setting $v=u_{k}^{\varepsilon}$ in (18) and using the coerciveness of $G_{\varepsilon}(\cdot, \cdot)$ and the orthonormality of $\left\{u_{k}^{\varepsilon}\right\}_{k}$ we find

$$
\begin{aligned}
G_{\varepsilon}\left(u_{k}^{\varepsilon}, u_{k}^{\varepsilon}\right) & =\int_{\Omega_{\varepsilon a}} a_{i j}^{\varepsilon} \frac{\partial u_{k}^{\varepsilon}}{\partial x_{j}} \frac{\partial u_{k}^{\varepsilon}}{\partial x_{i}} d x+\int_{\Omega_{\varepsilon b}} b_{i j}^{\varepsilon} \frac{\partial u_{k}^{\varepsilon}}{\partial x_{j}} \frac{\partial u_{k}^{\varepsilon}}{\partial x_{i}} d x+ \\
& +\varepsilon \int_{\Gamma_{\varepsilon}} h^{\varepsilon}\left[u_{k}^{\varepsilon}\right]^{2} d s=\lambda_{k}^{\varepsilon}, \quad \forall u^{\varepsilon} \in H_{\varepsilon} .
\end{aligned}
$$

As $\left\{\lambda_{k}^{\varepsilon}\right\}_{\varepsilon}$ is bounded, we find that

$$
\left\{u_{k}^{\varepsilon}\right\}_{\varepsilon} \text { is bounded in } H_{\varepsilon} \text {. }
$$

Using the previously obtained result together with inequalities (8)-(10), it follows that there exists $C>0$ such that

$$
\left|\nabla u_{k}^{\varepsilon}\right|_{L^{2}\left(\Omega_{\varepsilon a}\right)} \leq C, \quad\left|\nabla u_{k}^{\varepsilon}\right|_{L^{2}\left(\Omega_{\varepsilon b}\right)} \leq C, \quad\left|\left[u_{k}^{\varepsilon}\right]\right|_{L^{2}\left(\Gamma_{\varepsilon}\right)} \leq C .
$$

Applying to the properties of the two-scale convergence theory [1], a specific compactness result follows.

Theorem 3.1. There exist $\lambda_{k} \in \mathbb{R}_{+}^{\star}, u_{k}^{a} \in H^{1}(\Omega), u_{k}^{b} \in H_{0}^{1}(\Omega)$ and $\eta_{k}^{\alpha} \in$ $L^{2}\left(\Omega ; \widetilde{H}_{p e r}^{1}\left(Y_{\alpha}\right)\right), \alpha \in\{a, b\}$, such that the following convergences hold on some subsequence

$$
\begin{gathered}
\chi_{\alpha}^{\varepsilon} u_{k}^{\varepsilon} \stackrel{2 s}{\rightarrow} \chi_{\alpha} u_{k}^{\alpha}, \\
\chi_{\alpha}^{\varepsilon} \nabla u_{k}^{\varepsilon} \stackrel{2 s}{\rightarrow} \chi_{\alpha}\left(\nabla_{x} u_{k}^{\alpha}+\nabla_{y} \eta_{k}^{\alpha}(\cdot, y)\right), \\
\lambda_{k}^{\varepsilon} \rightarrow \lambda_{k},
\end{gathered}
$$

where $\chi_{\alpha}^{\varepsilon}: L^{2}\left(\Omega_{\varepsilon \alpha}\right) \rightarrow L^{2}(\Omega)$ and $\chi_{\alpha}: L^{2}\left(\Omega \times Y_{\alpha}\right) \rightarrow L^{2}(\Omega \times Y), \alpha \in\{a, b\}$, denote the straight prolongations with zero; sometimes they can be identified with the corresponding characteristic functions. 


\section{The homogenization process}

Passing (18) to the limit on the subsequence on which the convergences of Theorem 3.1 hold, we obtain like in [8]:

Lemma 4.1. For any $\varphi_{a} \in C^{\infty}(\bar{\Omega}), \varphi_{b} \in \mathcal{D}(\Omega)$ and $\psi_{\alpha} \in \mathcal{D}\left(\Omega ; C_{p e r}^{\infty}\left(Y_{\alpha}\right)\right)$, $\alpha \in\{a, b\}$, it holds

$$
\begin{gathered}
\int_{\Omega \times Y_{a}} a_{i j}\left(\frac{\partial u_{k}^{a}}{\partial x_{j}}+\frac{\partial \eta_{k}^{a}}{\partial y_{j}}\right)\left(\frac{\partial \varphi_{a}}{\partial x_{i}}+\frac{\psi_{a}}{\partial y_{i}}\right)+\int_{\Omega \times Y_{b}} b_{i j}\left(\frac{\partial u_{k}^{b}}{\partial x_{j}}+\frac{\partial \eta_{k}^{b}}{\partial y_{j}}\right)\left(\frac{\partial \varphi_{b}}{\partial x_{i}}+\frac{\psi_{b}}{\partial y_{i}}\right)+ \\
+\widetilde{h} \int_{\Omega}\left(u_{k}^{a}-u_{k}^{b}\right)\left(\varphi_{a}-\varphi_{b}\right)=\lambda_{k} \int_{\Omega \times Y} \chi_{a} u_{k}^{a} \varphi_{a}+\chi_{b} u_{k}^{b} \varphi_{b}
\end{gathered}
$$

where $\widetilde{h}$ is defined by

$$
\widetilde{h}=\int_{\Gamma} h(y) d s .
$$

Proof. For $\varphi_{\alpha}$ and $\psi_{\alpha}, \alpha \in\{a, b\}$ like in the hypotheses, we set $v$ in (18) as follows:

$$
v(x)=\left(\varphi_{a}(x)+\varepsilon \psi_{a}\left(x, \frac{x}{\varepsilon}\right), \varphi_{b}(x)+\varepsilon \psi_{b}\left(x, \frac{x}{\varepsilon}\right)\right) .
$$

We obtain

$$
\begin{aligned}
\lambda^{\varepsilon}\left(\chi_{a}^{\varepsilon} u^{\varepsilon}, \varphi_{a}\right) & +\lambda^{\varepsilon}\left(\chi_{a}^{\varepsilon} u^{\varepsilon}, \varphi_{b}\right)+\mathcal{O}(\varepsilon)=\sum_{\alpha \in\{a, b\}} \int_{\Omega_{\varepsilon \alpha}} \alpha_{i j}^{\varepsilon} \frac{\partial u^{\varepsilon}}{\partial x_{j}}\left(\frac{\partial \varphi_{\alpha}}{\partial x_{i}}+\frac{\partial \psi_{\alpha}}{\partial y_{i}}\right)+ \\
& +\varepsilon \int_{\Gamma_{\varepsilon}} h^{\varepsilon}\left(\gamma_{\varepsilon a} u^{\varepsilon}-\gamma_{\varepsilon b} u^{\varepsilon}\right)\left[\varphi_{a}-\varphi_{b}+\varepsilon\left(\psi_{a}-\psi_{b}\right)\right] .
\end{aligned}
$$

The proof is completed by following the same steps as in the proof presented in $[8]$.

In order to present the next results we have also to introduce the Hilbert space

$$
H:=\left[H^{1}(\Omega) \times H_{0}^{1}(\Omega)\right] \times\left[L^{2}\left(\Omega, \widetilde{H}_{p e r}^{1}\left(Y_{a}\right) \times L^{2}\left(\Omega, \widetilde{H}_{p e r}^{1}\left(Y_{b}\right)\right)\right],\right.
$$

endowed with the scalar product

$$
\begin{gathered}
\left(\left(\left(u_{a}, u_{b}\right),\left(\eta_{a}, \eta_{b}\right)\right),\left(\left(\varphi_{a}, \varphi_{b}\right),\left(\psi_{a}, \psi_{b}\right)\right)\right)_{H}=\sum_{\alpha \in\{a, b\}} \int_{\Omega} \nabla u_{\alpha} \nabla \varphi_{\alpha}+ \\
+\int_{\Omega}\left(u_{a}-u_{b}\right)\left(\varphi_{a}-\varphi_{b}\right)+\sum_{\alpha \in\{a, b\}} \int_{\Omega \times Y_{\alpha}} \nabla_{y} \eta_{\alpha} \nabla_{y} \psi_{\alpha} .
\end{gathered}
$$

Using density arguments, Lemma 4.1 yields: 
Theorem 4.1. $\lambda_{k} \in \mathbb{R}_{+}^{*}$ and $\left(\left(u_{k}^{a}, u_{k}^{b}\right),\left(\eta_{a}, \eta_{b}\right)\right) \in H \backslash\{0\}$ verify:

$$
\begin{gathered}
\int_{\Omega \times Y_{a}} a_{i j}\left(\frac{\partial u_{k}^{a}}{\partial x_{j}}+\frac{\partial \eta_{k}^{a}}{\partial y_{j}}\right)\left(\frac{\partial \varphi_{a}}{\partial x_{i}}+\frac{\psi_{a}}{\partial y_{i}}\right)+\int_{\Omega \times Y_{b}} b_{i j}\left(\frac{\partial u_{k}^{b}}{\partial x_{j}}+\frac{\partial \eta_{k}^{b}}{\partial y_{j}}\right)\left(\frac{\partial \varphi_{b}}{\partial x_{i}}+\frac{\psi_{b}}{\partial y_{i}}\right)+ \\
+\widetilde{h} \int_{\Omega}\left(u_{k}^{a}-u_{k}^{b}\right)\left(\varphi_{a}-\varphi_{b}\right)=\lambda_{k} \int_{\Omega \times Y}\left(\chi_{a} u_{k}^{a} \varphi_{a}+\chi_{b} u_{k}^{b} \varphi_{b}\right) \\
\forall\left(\left(\varphi_{a}, \varphi_{b}\right),\left(\psi_{a}, \psi_{b}\right)\right) \in H
\end{gathered}
$$

We can present now the main result of this paper.

Theorem 4.2. If $\left(\lambda_{k}^{\varepsilon}, u_{k}^{\varepsilon}\right)$ is a solution of (18) then the limits of the convergences (34)-(36), that is $\lambda_{k} \in \mathbb{R}_{+}^{*}$ and $\left(u_{k}^{a}, u_{k}^{b}\right) \in\left[H^{1}(\Omega) \times H_{0}^{1}(\Omega)\right] \backslash\{0\}$, put together a solution of the following effective eigenvalue problem:

Find $\left(\lambda_{k},\left(u_{k}^{a}, u_{k}^{b}\right)\right) \in \mathbb{R}_{+}^{*} \times\left[H^{1}(\Omega) \times H_{0}^{1}(\Omega)\right] \backslash\{0\}$ such that

$$
\begin{gathered}
G^{h o m}\left(\left(u_{k}^{a}, u_{k}^{b}\right),\left(\varphi_{a}, \varphi_{b}\right)\right)=\lambda_{k} \int_{\Omega} m u_{k}^{a} \varphi_{a}+(1-m) u_{k}^{b} \varphi_{b}, \\
\forall\left(\varphi_{a}, \varphi_{b}\right) \in H^{1}(\Omega) \times H_{0}^{1}(\Omega),
\end{gathered}
$$

where $m=\left|Y_{a}\right|$,

$$
\begin{gathered}
G^{h o m}\left(\left(u_{k}^{a}, u_{k}^{b}\right),\left(\varphi_{a}, \varphi_{b}\right)\right)= \\
\int_{\Omega} \hat{a}_{i j} \frac{\partial u_{k}^{a}}{\partial x_{j}} \frac{\partial \varphi_{a}}{\partial x_{i}}+\int_{\Omega} \hat{b}_{i j} \frac{\partial u_{k}^{b}}{\partial x_{j}} \frac{\partial \varphi_{b}}{\partial x_{i}}+\widetilde{h} \int_{\Omega}\left(u_{k}^{a}-u_{k}^{b}\right)\left(\varphi_{a}-\varphi_{b}\right),
\end{gathered}
$$

the effective coefficients $\hat{\alpha}_{i j}, \alpha \in\{a, b\}$, are defined by

$$
\hat{\alpha}_{i j}=\int_{Y_{\alpha}} \alpha_{i j}+\alpha_{i k} \frac{\partial e_{j}^{a}}{\partial y_{k}} d y, \quad \forall i, j \in\{1,2, \ldots, N\}
$$

and $e_{k}^{\alpha} \in \widetilde{H}_{p e r}^{1}\left(Y_{\alpha}\right), k \in\{1,2, \ldots, N\}$, is the unique solution of the localperiodic problem

$$
\begin{gathered}
-\frac{\partial}{\partial y_{i}}\left(\alpha_{i j} \frac{\partial\left(e_{k}^{\alpha}+y_{k}\right)}{\partial y_{j}}\right)=0 \quad \text { in } Y_{\alpha}, \\
\alpha_{i j} \frac{\partial\left(e_{k}^{\alpha}+y_{k}\right)}{\partial y_{j}} \nu_{i}=0 \quad \text { on } \Gamma .
\end{gathered}
$$

Moreover, there are no eigenvalues of the problem (44) except those obtained as limits of the homogenization process. 
Proof. The first assertion follows from Theorem 4.1 using standard homogenization procedures.

Next, suppose that there exists an eigenvalue of the problem (44), $\mu \in$ $\mathbb{R}_{+}^{*}, \mu \neq \lambda_{k}, \forall k \geq 1$, where $\left\{\lambda_{k}\right\}_{k}$ represents the eigenvalues of the problem (44) obtained as limits of the homogenization process. It follows that there exists $k$ such that $\lambda_{k}<\mu<\lambda_{k+1}$. Let $w=\left(w^{a}, w^{b}\right) \in\left[H^{1}(\Omega) \times H_{0}^{1}(\Omega)\right] \backslash$ $\{0\}$ be an eigenfunction associated to $\mu$.

Let us introduce $w^{\varepsilon} \in H_{\varepsilon}$, the unique the solution to the problem

$$
G_{\varepsilon}\left(w^{\varepsilon}, v\right)=\mu \int_{\Omega_{\varepsilon a}} w^{a} v+\mu \int_{\Omega_{\varepsilon b}} w^{b} v, \forall v \in H_{\varepsilon} .
$$

Setting $v=w^{\varepsilon}$ in the previous relation we find that

$$
G_{\varepsilon}\left(w^{\varepsilon}, w^{\varepsilon}\right) \leq C\left|w^{\varepsilon}\right|_{L^{2}(\Omega)} .
$$

Applying the coercivity property of $G_{\varepsilon}$, we find that the sequence $\left\{w^{\varepsilon}\right\}_{\varepsilon}$ is bounded in $H_{\varepsilon}$. Under these circumstances, there exists $w^{*}=\left(w_{a}^{*}, w_{b}^{*}\right) \in$ $H^{1}(\Omega) \times H_{0}^{1}(\Omega)$ such that

$$
P_{a}^{\varepsilon} w^{\varepsilon} \rightarrow w_{a}^{*} \text { in } H^{1}(\Omega) \text { and } P_{b}^{\varepsilon} w^{\varepsilon} \rightarrow w_{b}^{*} \text { in } H_{0}^{1}(\Omega) .
$$

Homogenizing the problem (49) we obtain

$$
\lim _{\varepsilon \rightarrow \infty} G_{\varepsilon}\left(w^{\varepsilon}, v\right)=G^{h o m}\left(w^{*}, v\right)=\mu(w, v)=G^{h o m}(w, v), \forall v \in H_{0}^{1}(\Omega),
$$

from which follows

$$
w=w^{*} \text { and } \int_{\Omega}\left(w^{\varepsilon}\right)^{2} \rightarrow \int_{\Omega} m\left(w^{a}\right)^{2}+(1-m)\left(w^{b}\right)^{2} \neq 0 .
$$

Next, we define $\hat{w}^{\varepsilon}=w^{\varepsilon}-\sum_{i=1}^{k}\left(\int_{\Omega} w^{\varepsilon} u_{i}^{\varepsilon}\right) u_{i}^{\varepsilon}$, where $u_{i}^{\varepsilon} \in H_{\varepsilon}$ is an eigenfunction associated to $\lambda_{i}$. We note that $\int_{\Omega} \hat{w}^{\varepsilon} u_{j}^{\varepsilon}=0, \forall j \leq k$, and hence

$$
G^{\varepsilon}\left(\hat{w}^{\varepsilon}, \hat{w}^{\varepsilon}\right) \geq \lambda_{k+1}^{\varepsilon} \int_{\Omega}\left(\hat{w}^{\varepsilon}\right)^{2} .
$$

As

$$
\int_{\Omega} w^{\varepsilon} u_{i}^{\varepsilon}=\int_{\Omega} \chi_{a}^{\varepsilon} P_{a}^{\varepsilon} w^{\varepsilon} u_{i}^{\varepsilon}+\int_{\Omega}\left(1-\chi_{a}^{\varepsilon}\right) P_{b}^{\varepsilon} w^{\varepsilon} u_{i}^{\varepsilon}, \quad \forall i \in\{1, \ldots, k\},
$$

the passage to the limit yields

$$
\int_{\Omega} w^{\varepsilon} u_{i}^{\varepsilon} \rightarrow \int_{\Omega} m w^{a} u_{i}^{a}+(1-m) w^{b} u_{i}^{b}=0,
$$


which obviously implies

$$
\int_{\Omega}\left(\hat{w}^{\varepsilon}\right)^{2} \rightarrow \int_{\Omega} m\left(w^{a}\right)^{2}+(1-m)\left(w^{b}\right)^{2} .
$$

Passing (49) to the limit in we finally get

$$
\begin{aligned}
G_{\varepsilon}\left(\hat{w}^{\varepsilon}, \hat{w}^{\varepsilon}\right) & \rightarrow G^{h o m}(w, w)=\mu \int_{\Omega} m\left(w^{a}\right)^{2}+(1-m)\left(w^{b}\right)^{2}< \\
& <\lambda_{k+1} \int_{\Omega} m\left(w^{a}\right)^{2}+(1-m)\left(w^{b}\right)^{2},
\end{aligned}
$$

which is in contradiction with (51), via (52).

Remark 4.1. The formulation of the effective two-temperature eigenvalue problem follows from Theorem (4.2):

Find $\lambda \in \mathbb{R}^{*}$ such that $\exists\left(u^{a}, u^{b}\right) \in\left[H^{1}(\Omega) \times H_{0}^{1}(\Omega)\right] \backslash\{0\}$ verifying

$$
\begin{gathered}
-\operatorname{div}\left(\hat{a} \nabla u^{a}\right)=m \lambda u^{a} \quad \text { in } \Omega, \\
-\operatorname{div}\left(\hat{b} \nabla u^{b}\right)=(1-m) \lambda u^{b} \quad \text { in } \Omega,
\end{gathered}
$$

and the boundary condition

$$
\hat{a}_{i j} \frac{\partial u^{a}}{\partial x_{j}} n_{i}=0 \text { on } \partial \Omega
$$

where $n$ is the outward normal on $\partial \Omega$.

Acknowledgements. The authors gratefully acknowledge partial support from the International Network GDRI ECO-Math.

\section{References}

[1] G. Allaire, Homogenization and two-scale convergence, S.I.A.M. J. Math. Anal. 23 (1992), 1482-151.

[2] J.L. Auriault, H. Ene, Macroscopic modelling of heat transfer in composites with interfacial thermal barrier, Internat. J. Heat Mass Transfer 37 (1994), 2885-2892.

[3] I. Babuška, J. Osborn, Eigenvalue Problems, Handbook of numerical analysis, vol II, Elsevier Science Publishers, North-Holland, 1991.

[4] H. Brezis, Analyse fonctionnelle. Théorie et applications, 1983, Dunod, Paris. 
[5] R. Bunoiu, C. Timofte, Homogenization of a thermal problem with flux jump, Networks and Heterogeneous Media 11(4):545-562 DOI: 10.3934/nhm.2016009 (2016), 1-22.

[6] D. Cioranescu and J. Saint-Jean-Paulin, Homogenization in open sets with holes, J. Math. Anal. Appl. 71 (2)(1979), 590-607

[7] P. Donato, S. Monsurrò, Homogenization of two heat conductors with interfacial contact resistance, Anal. Appl. 2 (3) (2004), 247-273.

[8] H.I. Ene, D. Poliševski, Model of diffusion in partially fissured media, Z.A.M.P. 53 (2002), 1052-1059.

[9] I. Gruais, D. Poliševski, Heat transfer models for two-component media with interfacial jump, Applicable Analysis, 96(2) (2015), 247-260.

[10] I. Gruais, D. Poliševski, Model of two-temperature convective transfer in porous media, Z.Angew. Math. Phys.(J. Appl. Math. Phys.), 68:143., https://doi.org/10.1007/s00033-017-0889-2 (2017), 1-10.

[11] H.K. Hummel, Homogenization for heat transfer in polycrystals with interfacial resistances, Appl. Anal., 75 (3-4) (2000), 403-424.

[12] S. Kesavan, Homogenization of elliptic eigenvalue problems, Appl. Math. and Optim. 5 (1979), Part I, 153-167, Part II, 197-216.

[13] R. Lipton, Heat conduction in fine scale mixtures with interfacial contact resistance, SIAM J. Appl. Math. 58 (1) (1998), 55-72.

[14] S. Monsurrò, Homogenization of a two-component composite with interfacial thermal barrier, Adv. Math. Sci. Appl., 13 (1) (2003), 43-63.

[15] D. Poliševski, The Regularized Diffusion in Partially Fractured Porous Media, Current Topics in Continuum Mechanics, Vol. 2, Ed. Academiei, Bucharest, 2003.

[16] D. Poliševski, R. Schiltz-Bunoiu, A. Stănescu, Heat transfer with firstorder interfacial jump in a biconnected structure, Bull. Math. Soc. Sci. Math. Roumanie 58(4) (2015), 463-473.

[17] M. Vanninathan, Homogenization of eigenvalue problems in perforated domains, Proceedings of the Indian Academy of Sciences. Mathematical Sciences 90(3) (1981), 239-271. 